\title{
Laboreal
}

Volume $8 \mathrm{~N}^{\circ} 1$ | 2012

Género, Atividades e Saúde

\section{¿Una profesión "women friendly" ? : lo que está en juego en la organización del trabajo de docentes} francesas y españolas

Uma profissão "women friendly" ? : o que está em jogo na organização do trabalho de professoras francesas e espanholas

Une profession "women friendly" ? : les enjeux de l'organisation du travail des enseignantes françaises et espagnoles

A "women friendly" profession? : the repercussions of the French and Spanish women teachers' work organisation

Julie Jarty

\section{OpenEdition}

\section{Journals}

Edición electrónica

URL: http://journals.openedition.org/laboreal/7161

DOI: 10.4000/laboreal. 7161

ISSN: 1646-5237

Editor

Universidade do Porto

Referencia electrónica

Julie Jarty, « ¿Una profesión "women friendly" ? : lo que está en juego en la organización del trabajo de docentes francesas y españolas », Laboreal [En línea], Volume 8 No1 | 2012, Publicado el 01 julio 2012, consultado el 11 octubre 2019. URL : http://journals.openedition.org/laboreal/7161 ; DOI : 10.4000/ laboreal.7161

Este documento fue generado automáticamente el 11 octubre 2019

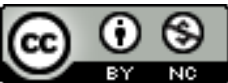

Laboreal está licenciado com uma Licença Creative Commons - Atribuição-NãoComercial 4.0 Internacional. 


\section{¿Una profesión "women friendly" ? : lo que está en juego en la organización del trabajo de docentes francesas y españolas}

Uma profissão "women friendly" ? : o que está em jogo na organização do trabalho de professoras francesas e espanholas

Une profession "women friendly " ? : les enjeux de l'organisation du travail des enseignantes françaises et espagnoles

A "women friendly" profession?: the repercussions of the French and Spanish women teachers' work organisation

Julie Jarty

\section{NOTA DEL EDITOR}

Manuscrito recibido en : Febrero/2012

Aceptado tras peritaje : Mayo/2012

\section{Introducción}

1 Aunque algunos estudios recientes señalan las experiencias de articulación de las temporalidades profesionales y extra-profesionales como una clave de lectura indispensable para la comprensión de las experiencias laborales de las mujeres (Meda \& Perivier, 2007; Tobío Soler, 2005), siguen siendo raros, tanto en Francia como en España, los trabajos acerca de la profesión docente que integran tal perspectiva. Hay que decir que los estudios de género han prestado poca atención a una profesión cuya importante proporción de mujeres $\left.{ }^{1}\right]$ parece conferir a la misma un estatuto de 
"remanso de igualdad" (Cacouault-Bitaud, 2007 ; Rovira, 1993). Por un lado, habría menos discriminaciones y manifestaciones de sexismo que en otros oficios : la función pública preservaría a sus asalariados y asalariadas de las desigualdades más notorias (principalmente en cuanto concierne a las oposiciones de acceso o al progreso en la carrera profesional en función de la antigüedad). Por otra parte, los tiempos de la actividad docente serían particularmente compatibles con las responsabilidades domésticas y familiares que las mujeres siguen asumiendo todavía de manera muy mayoritaria en ambos países (Carrasquer, Oto, Torns, Tejero \& Romero, 1998 ; Heinen, Hirata \& Pfefferkorn, 2009; Torns, 2005). A un lado y otro de los Pirineos se habla de una profesión family friendly, e incluso women friendly.

2 Sin embargo, este impasse resulta tanto más sorprendente cuanto que, sobre un trasfondo de "crisis" [2] de la profesión (Lantheaume \& Helou, 2008) y de incremento al alza de los indicadores de estrés vinculados a la misma (absentismo, agotamiento, depresiones múltiples, jubilación anticipada...) en la mayoría de países occidentales (ETUCE, 2011), la organización del tiempo de trabajo aparece como un factor social y de salud fundamental. Por supuesto, la intensidad del trabajo docente, la multiplicación de obligaciones, el número creciente de efectivos en las clases e incluso el comportamiento cada vez más ingobernable de algunos alumnos son reconocidos como causas del carácter penoso que va adquiriendo la profesión, por no decir de la degradación de su entorno (ETUCE, 2011). Pero, en esa profesión y de un modo específico, ¿no son acaso las mujeres objeto de tensiones derivadas de la división sexual del trabajo? En cualquier caso, eso es lo que sugieren algunos trabajos recientes, que analizan la actividad docente bajo una óptica de género en el seno de distintos contextos sociales. Esos estudios subrayan la singularidad de las experiencias femeninas frente a los insistentes llamamientos a la "conciliación" (Caroly \& Messing, 2011 ; Jarty, 2009, 2011 ; Riel \& Messing, 2011). Las dificultades para compatibilizar esferas profesionales y familiares motivarían abandonos anticipados del trabajo en Quebec, Canadá (Butt \& Lance, 2005) y en Francia (Cau-Bareille, 2011). Esa pista debería ser tomada muy en serio en la medida que, si bien existen pocos estudios que nos faciliten información acerca de la distribución de los factores de estrés en función de la categoría de sexual, algunas investigaciones muestran que las mujeres enseñantes son más proclives a la fatiga y a la depresión que sus homólogos masculinos (Castro Posada, 2005 ; Houlfort \& Sauvé, 2010 ; Matud Aznar, Garcia Rodriguez \& Matud Aznar, 2002).

3 Este artículo propone un enfoque sociológico de los contextos y experiencias reales de trabajo de las enseñantes francesas y españolas, a la luz de un análisis de los tiempos de actividad ampliado a los marcos y contextos de vida extra-profesionales. Por otro lado, se trata también de interrogarse acerca del modo en que se organizan las actividades, de manera distinta si se trata de hombres o mujeres, así como de aprehender sus posibles consecuencias en cuanto a la relación entre trabajo y salud se refiere. Tras definir los marcos metodológicos y conceptuales sobre los que se basa este artículo, se desarrollará en tres partes. La primera estará dedicada a poner en perspectiva la especificidad de la organización temporal del trabajo de los enseñantes a tenor de las normas y las "culturas de género" imperantes en el contexto organizativo de cada país. El estudio proseguirá a través de una segunda parte, focalizada sobre el análisis del reparto sexuado de los tiempos personales (de ocio y particular), concebido en estrecha vinculación con las prácticas anteriormente identificadas. Finalmente, esos resultados 
darán pié a una reflexión sobre las consecuencias del ritmo (sexuado) del tiempo de los y las enseñantes desde el punto de vista de las experiencias de salud laboral.

\section{Marco metodológico y analítico}

\subsection{Dispositivo de investigación}

Este artículo se apoya sobre los resultados de una tesis de sociología titulada "El empleo, la familia y la articulación de los tiempos vitales entres las y los enseñantes de secundaria. Una comparación entre Francia y España". Dicha tesis se desarrolló en varias fases. En primer lugar, se llevó a cabo una investigación de campo, una cartografía de los contextos francés y español, con objeto de aprehender las normas de actividad profesional dominantes, y luego un estudio comparativo de la estructuración de la profesión docente en ambos países. Desde un punto de vista cualitativo, la investigación se basa sobre distintos dispositivos de encuesta. El principal consistió en la realización de 75 entrevistas biográficas con enseñantes de ambos sexos [3] en establecimientos franceses y españoles de segundo grado, llevadas a cabo sobre la base del voluntariado. Todas y todos trabajan en el sector público. Tenían entre 29 y 61 años cuando tuvieron lugar las entrevistas y presentaban situaciones familiares variadas. La opción de una franja de edad amplia se justificaba por la necesidad de captar las diferencias intergeneracionales en lo referente a las representaciones y a las prácticas en el ejercicio de la profesión y a la división sexual del trabajo. Todas las entrevistas han sido transcritas íntegramente y analizadas según unas pautas de lectura establecidas en estrecha relación con los temas abordados a lo largo de la conversación: la socialización primaria, la opción por esta profesión, el desarrollo de la carrera, la organización de la vida familiar y doméstica, la gestión del tiempo libre.

5 Estas entrevistas se han añadido, por un lado, a una fase de observación efectuada paralelamente a las conversaciones que tuvieron lugar en el seno de los establecimientos escolares. Se trataba de aprehender mejor los lenguajes, códigos y prácticas profesionales tal como se forjan en el seno del entorno profesional "formal". Por otro lado, fueron analizados 17 "cuadernos temporales" rellenados por las y los propios docentes entrevistados. Esos cuadernos tenían la forma de un diario íntimo en cuyas páginas se consignaba, a lo largo de toda una semana, cada detalle de la jornada. Se pretendía así afinar el análisis de los "espacios-tiempos" en lo concerniente a la realización del trabajo docente, penetrando en un entorno más "informal" de la realización de dicho trabajo : la esfera privada.

\subsection{Postura teórica}

6 En la intersección de los enfoques interaccionistas y de las perspectivas feministas, un cierto número de estudios han demostrado ya la pertinencia de las investigaciones centradas en un nivel meso de análisis; es decir, integrando un estudio de las normas y valores difundidos en el seno de las distintas profesiones, para comprender las experiencias laborales de las mujeres en las sociedades occidentales contemporáneas (Fusulier, 2011; Le Feuvre, 2010). Desde el punto de vista de género, esta perspectiva brinda nuevas pistas de reflexión para pensar las desigualdades de sexo por cuanto a la articulación de los tiempos sociales se refiere. Así, las condiciones objetivas de la 
profesión (modos de gestión de la mano de obra empleada en ella, constricciones temporales y espaciales de su ejercicio, etc.) constituyen otros tantos elementos que inciden sobre la manera en que las pertenencias sexuadas pesan sobre las prácticas concretas de organización cotidiana del trabajo. De un sector de actividad a otro, pero también de un país a otro en el seno de un mismo sector de actividad, se forjan así determinadas "culturas de género", maneras de hacer legítimas, percibidas como ideales, en función del sexo al que se pertenezca. Aproximándose a la noción de "regímenes de género" utilizada por Connell (1987), tales "culturas" imprimen su sello particular sobre las maneras de comprometerse con el trabajo. Par estudiar esas normas dominantes, el presente artículo se apoya, siguiendo los pasos de la sociología de las relaciones sociales de sexo, sobre una conceptualización específica del trabajo.

7 En efecto, mientras que la aprehensión sociológica del trabajo, dominante durante mucho tiempo, se focalizaba sobre las actividades productivas remuneradas, los estudios de género han puesto de relieve que el trabajo comporta, tanto para las mujeres como para los hombres, una amplia gama de actividades (remuneradas y no remuneradas). Indiscutiblemente, este postulado permite aclarar la singularidad de las historias de la vida profesional de las mujeres, de su relación con el trabajo y del desgaste que puede derivarse de su ejercicio. De hecho, a ellas incumbe siempre de modo prioritario la "conciliación", generalmente más conflictiva que armoniosa, de las esferas profesionales y familiares (Durán, 2006 ; Junter-Loiseau, 1999; Heinen et al., 2009). Se trata pues, al cabo, de considerar la importancia de los distintos marcos y contextos de trabajo - profesionales y extra-profesionales, trabajo y "fuera del trabajo" - para captar con mayor sutileza las "recíprocas influencias" de los tiempos de vida (Gadbois, 1975), así como la organización temporal sexuada que acaba instalándose en tal o cual grupo profesional (Messing y Ostling, 2006).

Esa postura se solapa con las investigaciones en el campo de la psicología del trabajo (Curie, 2002 ; Almudever, Croity-Belz, Hajjar \& Fraccaroli, 2006) que proponían "abarcar a la vez" diferentes espacios de vida - "subsistemas", por retomar el vocablo que utilizan : profesional, familiar y de ocio. En un reciente análisis de la relación con el trabajo por parte de maestras de maternal en Francia, Cau-Bareille invita a reconsiderar la pertinencia de dicha perspectiva para vislumbrar con mayor precisión los fenómenos, hasta la fecha poco estudiados, de desgaste prematuro en el seno de profesiones fuertemente feminizadas (Cau-Bareille, 2011). El interés de esta perspectiva reside en su capacidad para mostrar que la atención prestada a los demás, así como los riesgos de desaparición del tiempo de la persona "para sí misma" que comporta "la gestión ordinaria de sus vidas" (Haicault, 1984), inciden en la salud de las mujeres que permanecen en el mercado de trabajo después de ser madres (Burton-Jeangros \& Camenisch, 2006). A partir de ahí, siguiendo a Le Feuvre, conviene "romper con cierto número de automatismos analíticos, en particular con aquél que consiste en aprehender el trabajo remunerado como un fenómeno "amenazador" para la salud de los individuos, considerando que las restantes esferas de la vida social serían necesariamente aptas para "protegerla"” (Le Feuvre, $2011: 121$ ). 


\section{El trasfondo de los tiempos de la actividad docente en Francia y en España}

9 La profesión docente aparece, a través de las evoluciones históricas habidas a lo largo del siglo XX y de su representación como un oficio típicamente femenino, como un sector de empleo (relativamente) feminizado (Cacouault-Bitaud, 2007). Es el caso, tanto en Francia como en España, aunque el proceso de feminización se haya producido siguiendo ritmos discordantes: sólo a principios del nuevo siglo se cuentan tantos hombres como mujeres por lo que respecta a la enseñanza secundaria en España, mientras que eso era así desde finales de la década de 1970 al otro lado de los Pirineos. Las características de la profesión, particularmente por cuanto se trata de la transmisión de ideas (educación y formación de las jóvenes generaciones) y temporales (la proximidad de los calendarios profesionales con los de los niños), la acercan a los llamados "oficios de mujeres" que aseguran una importante disponibilidad para la vida familiar (Perrot, 1987). Es necesario subrayar que, en ambos países, los y las enseñantes de secundaria gozan de unos horarios que les permiten cierta proximidad con los de sus hijos (en caso de que los tengan) - concretamente, por lo que respecta a las vacaciones escolares. Sin embargo, esta autonomía temporal sigue siendo distinta en los dos contextos sociales. ¿Cuáles son las consecuencias de tal disparidad desde el punto de vista de la organización temporal concreta establecida por las enseñantes y de las relaciones de género?

\subsection{Torsiones y tensiones de las temporalidades "público-privado" entre las francesas}

\subsubsection{Del sentimiento de libertad a la maleabilidad de los ritmos de trabajo...}

10 En Francia, mucho más que en España, la figura profesoral dominante conforta las prácticas de contracción del tiempo que se pasa en los establecimientos escolares (limitado a la duración de las clases y a las reuniones obligatorias), aunque las recientes transformaciones de la profesión van en el sentido de un mayor volumen de trabajo colectivo: "en el colegio no hago gran cosa como trabajo individual, las preparaciones, las correcciones (...), nunca se trata de tareas muy profundas”, explica Annie, de 50 años. Así, podemos observar la existencia de prácticas ampliamente admitidas de negociación del reparto de las horas de careo con los alumnos (15 para los agregados, 18 para los certificados), con adecuación a las prioridades profesionales - según la percepción de su grado de competencia, por ejemplo -, pero también extra-profesionales de cada cual - según la dedicación, más o menos intensa, a la vida familiar o asociativa (Jarty, 2009). Generalmente, las direcciones intentan responder "lo mejor posible" a esas demandas dentro de los límites objetivos de las concordancias de los distintos horarios.

11 Resulta de ello un importante traslado de tareas profesionales a espacios tradicionalmente poco asociados a la materialidad del trabajo productivo y a una (aparente) libertad de gestión del tiempo : "es modulable", responde Emmanuelle cuando se le pregunta acerca del reparto espacial de su actividad; "disponemos libremente de nuestro tiempo", resume Diane.

12 El carácter limitado de los tiempos de presencia "obligatoria" en los establecimientos escolares permitido a las mujeres (y casi exclusivamente a ellas) ha contribuido al 
asentamiento de una "cultura de género" altamente diferenciadora por lo que respecta a las prácticas de gestión del tiempo. En general, las enseñantes desarrollan estrategias de calcado de sus calendarios profesionales y sus calendarios domésticos: "si quieres saber mis horarios, se corresponden con los de mis hijos", resume Marie, profesora de letras de 33 años y madre de dos niños. Las jefas y jefes de establecimiento entrevistados suscriben con clemencia y compasión a esas solicitudes de "adecuación de los horarios a la disponibilidad de las mamás", según su propia expresión. De manera igualmente recurrente, las encuestadas - mucho más que sus homólogos masculinos - explican que trasladan su trabajo de preparación de los cursos y de corrección a su tiempo supuestamente "libre" o de descanso, es decir a los fines de semana o a las noches : "me tomo un cierto tiempo al final de la tarde, pero lo recupero por la noche", anota Sarah, a la una de la madrugada, en su cuaderno. Esta observación confirma los resultados de Cacouault-Bitaud (2003) y de Cau-Bareille (2011), subrayando el hecho de que los hombres pasan un mayor tiempo en los establecimientos, mientras que las mujeres utilizan mucho más sus fines de semana y sus noches para preparar sus clases y corregir ejercicios.

Más aún: muchas son las que explican tácticas de superposición de actividades profesionales y extra-profesionales destinadas a rentabilizar el menor espacio-tiempo: "cuando estás en el coche, puedes trabajar", racionaliza Jeanne, 40 años, acostumbrada a corregir ejercicios en el coche al mismo tiempo que supervisa los deberes de uno de sus hijos y el otro sigue su curso de música. La vida familiar estructura e invade ampliamente el campo de la actividad profesional de las enseñantes francesas; su capacidad para gestionar de modo simultáneo las exigencias de distintas esferas de vida aparece como una competencia indispensable, tal como lo ilustran perfectamente las palabras de Maryse :

"Yo trabajaba durante todas las vacaciones, ya me había acostumbrado a trabajar rodeada de toda una tribu de niños, pero conseguía concentrarme y trabajar". Maryse, 58 años, Francia

\subsection{2 ...al malestar inducido por una sobreinversión en el trabajo de cuidados}

15 Sin embargo, tales condiciones de trabajo pueden revelarse problemáticas y agotadoras. Un sentimiento de insatisfacción, incluso de desánimo, se desprende con mucha frecuencia de las entrevistas con las enseñantes francesas madres de niños de baja edad. Algunas de ellas señalan los aspectos retorcidos de esa flexibilidad y esa autonomía temporal :

\footnotetext{
"Es verdad que, cuando eres enseñante, el aspecto positivo consiste en que pasas mucho tiempo en casa, y el aspecto negativo es que... durante todo ese tiempo, deberías estar trabajando, y yo me siento desbordada, porque cuando estoy con los niños me resulta difícil trabajar y eso es duro. Es verdaderamente duro porque me siento atrapada, no consigo liberar suficiente tiempo para trabajar. Desde la llegada de M. (nombre de su segundo hijo), me veo obligada a trabajar todas las noches y a levantarme muy temprano por la mañana para currar. Lo ideal sería que llegase a organizarme mejor, pero bueno... Sí, cuando tienes niños, trabajar en casa es una verdadera trampa". Agnès, 34 años, Francia
}

16 Si la actividad profesional puede verse amenazada al realizarse de manera precipitada, el riesgo de estrés resulta también evidente. Los "cuadernos temporales" se revelan 
como una herramienta formidable para captar y objetivar el estrés y el agotamiento de las enseñantes madres de niños pequeños. Con cierto humor, Sabine, profesora de matemáticas de 38 años y madre de dos niños (de 5 y 8 años respectivamente cuando tuvo lugar la entrevista), comenta al final de su cuaderno: "Tenía la impresión de estar siempre corriendo, ahora sé que no se trata de una simple impresión". Después de nuestra entrevista, Sandrine me contactó para decirme que no conseguía rellenar su cuaderno, explicándome que ese ejercicio le resultaba muy penoso y le devolvía la imagen de una vida convertida en una "carrera contra reloj" y dedicada exclusivamente a los otros. Sarah, de 33 años, joven mamá de un niño de un año, escribe después de una velada corrigiendo ejercicios hasta la una de la madrugada que "ya no aguanta más". Y Claire, que ha calcado su horario sobre el de sus hijos, se pregunta: "El miércoles, ¿es verdaderamente un día de descanso ?". Más que la actividad docente propiamente dicha, es el imperativo de una disponibilidad permanente hacia los demás lo que agobia a las mujeres que constituyen la población estudiada. La "doble (o triple) presencia" de las mujeres parece pues pesar también sobre las profesiones feminizadas, presuntamente más compatibles con la esfera familiar.

\subsection{El alejamiento del modelo de la madre enseñante siempre disponible por parte de las españolas : ¿una (des)ilusión ?}

\subsubsection{Una autonomía profesional (más) controlada}

17 En el contexto español, el dominio del tiempo de trabajo se realiza sobre un trasfondo de obligaciones temporales más ajustadas puesto que la presencia obligatoria en los establecimientos escolares no se limita a las horas lectivas, dentro de la clase. En ese caso, se trata de asegurar una presencia para vigilar el patio de recreo, substituir a los colegas ausentes, hacerse cargo de las tareas administrativas o pastorales que forman parte del trabajo docente [4]. Además, la colaboración en las actividades colegiales de dirección (sinónimo de una mayor presencia en los establecimientos) es parte integrante de la profesionalidad docente española. Todas esas especificidades levantan barreras temporales más rígidas que en Francia, aunque la profesión se caracterice por una flexibilidad temporal moderada, asociada a una toma en consideración de las preocupaciones familiares sensiblemente más elevada que en las profesiones del sector privado [5]. De hecho, "apañarse" un horario adaptado a las exigencias personales constituye una preocupación menor que en Francia. Los y las enseñantes disponen de espacios de trabajo específicos (despachos generalmente compartidos) para ejercer las actividades que envuelven la enseñanza propiamente dicha en clase (preparación de los cursos, ejercicios o exámenes; elaboración de proyectos pedagógicos, correcciones, reuniones, etc.). Un universo "orientado a la familia" se desprende de los discursos recogidos : distintas encuestas nos han evocado la posibilidad de volver más temprano a casa si eso no altera los horarios de clase, o la presencia ocasional de un niño que se ha puesto enfermo en el fondo del aula : "Recuerdo estar en clase durante un examen con el bebé allí al lado", explica Lourdes, de 61 años. Los relatos que evocan la importancia de su autonomía en la realización del trabajo y el carácter modulable del grado de "compromiso personal" (Bidet, 2011b) en las labores de gestión de la vida del establecimiento reflejan los contornos de una cultura profesional caracterizada por una autonomía controlada del trabajo. 
18 Así, en un país marcado por el alto de nivel de conflictividad de la interfaz "trabajo/ familia" a causa de la escasa legitimidad del estado para intervenir en la vida privada, y por lo tanto con una débil implicación de los servicios públicos en dirección de la pequeña infancia (Letablier, 2009), ser enseñante comporta ciertas facilidades para los padres por cuanto se refiere a articular vida profesional y vida familiar, pero no basta para evitar las tensiones inherentes a ello.

\subsubsection{Prácticas sexuadas más sutiles}

19 En España, la implicación espacio-temporal más elevada del personal docente en los institutos favorece cierta proximidad entre los calendarios profesionales femeninos y masculinos. La rentabilización del tiempo de permanencia en los establecimientos escolares, la utilización de los despachos disponibles (frecuentemente compartidos con algunos colegas de la misma disciplina), participan de una estrategia de compartimentación de los "espacios-tiempos" profesionales y personales y de una "cultura de género" más transgresiva desde el punto de vista del "sistema sexo/ género" (Kergoat, 2000).

"Aprovecho mucho el tiempo que tengo en el Instituto para hacer el máximo, o sea que preparo más en el Instituto. Mi casa es mi casa, mi trabajo es mi trabajo, ¿vale ?". Martina, 55 años, España

20 Estas prácticas compartimentadas no son inmutables, algunas fases de la vida profesional o familiar pueden requerir la flexibilización de esa organización. Con todo, sigue siendo una "consigna" entre las enseñantes españolas - algo poco visible en cuanto se refiere a las francesas, que reivindican, muy al contrario, las ventajas inherentes a la flexibilidad en el ejercicio de su profesión. Contrariamente a lo que hemos podido observar en Francia, la desconexión de las esferas profesionales y personales se presenta como una verdadera estrategia de regulación del desbordamiento y de la contaminación, por parte del tiempo que corresponde al trabajo asalariado, de los otros tiempos de la vida social.

21 Esta menor diferenciación sexuada en la organización del trabajo se ve reforzada por la presencia de "pioneras" (Marry, 2004) que se distinguen de la mayoría de las otras españolas al adoptar prácticas profesionales (pero también conyugales) atípicas (formación de parejas con consortes "participativos" tan diplomados como ellas, por ejemplo). Haciéndose eco de la postura "viril" descrita por Le Feuvre (2007), adoptan comportamientos que tienen que ver con las actitudes masculinas, ampliando sus horarios de estancia en el Instituto hasta avanzada la tarde o saliendo "con las mujeres de la limpieza a las nueve de la noche" (Elvira, 55 años), de tal modo que reducen su participación en las tareas domésticas recurriendo a una ayuda remunerada "de lo contrario sería demasiado duro" (Bibiana, 49 años). La mayoría ha limitado la talla de su familia y se ha limitado a tener un solo hijo "porque trabajando los dos era complicado", pero también porque sabían que "la carga (asociada al cuidado de otro niño) hubiese recaído de nuevo sobre mi", añaden respectivamente Elvira y Bibiana. Entre la joven generación, menos comprometida en las prácticas de sobreinversión profesional, la subcontratación del trabajo doméstico se integra también perfectamente en su "gestión de la familia". Permite soslayar la disputa en torno a las tareas domésticas, al tiempo que flexibiliza la continuidad entre tiempo de trabajo y tiempo personal : "Es un tema que hemos resuelto pagando". Ese resultado apunta en la misma dirección que toda una 
serie de investigaciones españolas que ponen de relieve la banalización del recurso a una ayuda remunerada por parte de las jóvenes parejas en que ambos miembros ejercen una actividad profesional (Torns \& Moreno, 2008 ; Fernández Cordon \& Tobio Soler, 2005).

No obstante, las españolas siguen gestionando generalmente la carga mental de la organización de las actividades. Como explica Lourdes, "el "prever situaciones", eso el hombre no lo tiene incorporado, en cambio nosotras si". De manera menos exacerbada que en Francia, las prácticas de superposición de los tiempos de vida se insinúan también entre aquellas que acumulan responsabilidades domésticas y familiares: "corregir... mucho en el metro, porque en casa no puedo trabajar" (Ana, 39 años). Pero se trata sobre todo de rentabilizar los tiempos intersticiales, es decir entre dos actividades principales, como los tiempos de transporte y de desplazamiento. Estos últimos son entonces utilizados para finalizar un trabajo y no interferir en la vida de los demás :

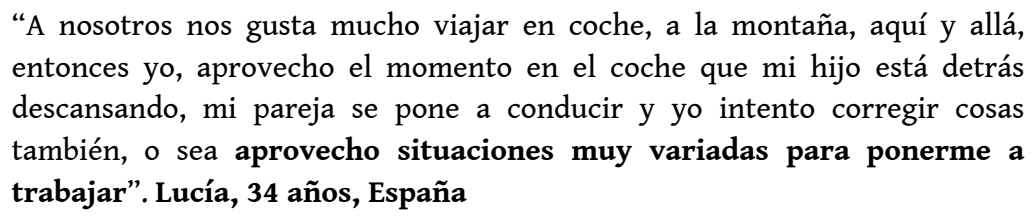

El objetivo sigue siendo el mismo : evitar las repercusiones de la vida profesional sobre su pareja y sobre los niños. En eso las "preocupaciones" de francesas y españolas coinciden. Pero las formas concretas de esta resistencia de los cimientos de la división sexual del trabajo se articulan estrechamente con los talantes profesionales singulares, que inciden sobre los ritmos en la organización del trabajo docente. La pretensión de este texto es comprender las repercusiones de tales prácticas sexuadas en la esfera del tiempo de ocio.

\section{La gestión del tiempo "libre" : un tope a la indiferenciación de las experiencias docentes}

La permeabilidad psicológica entre las temporalidades profesionales y extraprofesionales de las personas constituye un fenómeno que ha sido descrito con mucha anterioridad, en particular por parte de los ergónomos (Teiger, 1980), reconociendo las interacciones entre el trabajo y el tiempo libre. Estas últimas merecen una atención singular en un estudio acerca de las temporalidades docentes. En efecto, el trabajo docente reviste la especificidad de que una parte de sus tareas se realiza con mayor o menor intensidad en función del sexo, la disciplina o el país estudiado, en los "espaciostiempos" privados. Es el caso de la formación personal o de la profundización de la cultura que se realiza también durante los ratos de ocio, pues cada nuevo descubrimiento, cada lectura, es susceptible de alimentar un curso ulterior. Eso puede dar lugar a la sensación, por lo que respecta a los enseñantes de ambos sexos y países, de que están trabajando en todo momento (Jarty, 2010). Pero esa percepción de ausencia de tiempo para sí mismos, ¿no adopta acaso otras formas en el caso de las enseñantes? Pues, del mismo modo que el trabajo profesional no se detiene "en el umbral de la puerta" de casa, el tiempo dedicado a los demás (el tiempo de cuidados, por ejemplo) no se compartimenta tampoco tan fácilmente con respecto al tiempo libre "autónomo", el tiempo consagrado a sí mismo, en particular por lo que concierne a las 
mujeres (Deem, 1986). En ese punto, las experiencias de las francesas y las españolas se diferencian relativamente poco.

\subsection{Tiempo libre... para los demás}

La mayoría de enseñantes entrevistadas presentan una utilización del tiempo individual reducida a su mínima expresión: "no, no, no practico ningún deporte, he abandonado todo eso" (Marie, 33 años), "bueno, precisamente es eso lo que falla" (Claire, 33 años), "mis actividades, francamente, se reducen a cero, en fin prácticamente a cero, a decir verdad" (Emmanuelle, 29 años). Sólo las mujeres que reciben una ayuda activa por parte de su entorno familiar (puede tratarse de los abuelos cuando no ha habido movilidad geográfica o bien, aunque no sea frecuente, del marido) escapan a ese fenómeno de casi desaparición del tiempo libre.

La llegada del primer hijo es muchas veces evocada como el desencadenante de un giro radical en la manera de disponer de un tiempo "para sí mismo", que se transforma en tiempo "para los demás". Muchas presentan el paso al estatuto de madre como una transición brusca, que supone pasar de unas prácticas sociales orientadas hacia el exterior a unas prácticas esencialmente circunscritas a la familia. No cabe duda, se trata de una "travesía del desierto" por lo que respecta al tiempo de "ocio autónomo". La dedicación a los demás y la adaptación a "los ritmos del niño", según la propia expresión de Emmanuelle, emergen entonces como un rasgo estructurador de las experiencias personales de las enseñantes. Los miércoles por la tarde se asemejan a una carrera contra reloj. Se trata de ocuparse de los niños, a la vez para asegurar su desarrollo personal (lo que comporta con frecuencia que participen en un montón de actividades culturales y deportivas), pero es necesario también liberar algunos ratos para avanzar en el trabajo :

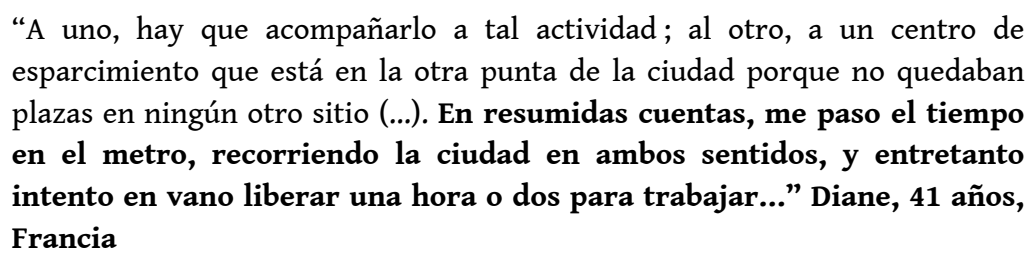
esparcimiento que está en la otra punta de la ciudad porque no quedaban plazas en ningún otro sitio (...). En resumidas cuentas, me paso el tiempo en el metro, recorriendo la ciudad en ambos sentidos, y entretanto intento en vano liberar una hora o dos para trabajar..." Diane, 41 años, Francia

Percibidos como momentos privilegiados para la familia, los fines de semana se estructuran en torno a los tiempos de ocio y de distensión con los niños. Sin embargo, las encuestas describen regularmente un estrés permanente durante esos períodos, en que el trabajo tampoco las abandona por completo. Una vez más, la permeabilidad de los tiempos profesionales y personales refuerza el carácter difuso del tiempo de trabajo de las enseñantes francesas. Este aspecto de la organización temporal no siempre deja de operar durante las vacaciones. Consagrándose prioritariamente a su familia, son muchas las que no consiguen "desconectar" durante esos períodos. Ése es en particular el caso de las profesoras de disciplinas literarias que, con frecuencia, se ven obligadas a corregir fajos de redacciones que han ido amontonándose a lo largo de las semanas anteriores.

En el caso de las españolas de la "generación de la transición" y la generación precedente (Astelarra, 2005), su fuerte consciencia de género les permite elaborar un discurso lúcido sobre el carácter sexuado del reparto de los tiempos de actividad, como lo hace, por ejemplo, Bibiana : 
“Tenía tiempo libre. Pero, por comparación, él (su ex marido) tenía el doble, ¡en la misma profesión !, ¡el mismo trabajo !” Bibiana, 49 años, España mujeres y las tentativas de preservar unos mínimos son numerosas. En esos casos, la contracción del tiempo personal constituye una práctica bastante corriente por parte de las enseñantes, de tal modo que interfiere lo menos posible con la vida de los hijos y con la de la pareja. Algunas de nuestras entrevistadas escogen la solución del club de gimnasia durante la pausa del desayuno, entre dos horas de clase: "Este curso, algunas colegas hemos decidido motivarnos para ir juntas al gimnasio. (...) Comemos en quince minutos, salimos a toda pastilla hacia el gimnasio, nos damos una ducha rápida y a las dos estamos frente a nuestros alumnos", bromea Emmanuelle. El objetivo es siempre el mismo : no perturbar los horarios de la pareja, conservar la forma y cierta apariencia, cueste lo que cueste: "Es algo vital, en fin... jHay que mantenerse de algún modo cuando has superado los cuarenta $y$ has tenido un par de niños!", explica Jeanne.

Según una lógica de disponibilidad permanente en relación con su familia, el tiempo personal de las francesas se torna secundario, por no decir accesorio. Podríamos añadir que esas prácticas con aire de buenos propósitos resultan a veces difíciles de llevar a cabo. Marie evoca el abono de un año que adquirió en un club de fitness donde, finalmente, "nunca llegó a poner los pies". Dando fe de los raros momentos en que se encuentra a solas consigo misma, Jeanne comenta su aseo matutino en el "cuaderno de tiempos": "Un momento agradable, aunque sea temprano; uno de esos raros momentos en que puedo ocuparme de mi misma". (Jeanne nos había contado, en efecto, durante la entrevista que se levantaba media hora antes que sus hijos para "estar a punto"). La 
lectura de su cuaderno revela tiempos de ocio y de descanso íntimamente ligados al trabajo doméstico (paseo con los niños... para ir a buscar el pan) o a las tareas domésticas (ir de compras a las grandes superficies especializadas, etc.).

La superposición de los tiempos sociales que se observa entre las enseñantes parece entonces interminable, pero la ausencia de compartimentación de las prácticas de ocio refuerza el sentimiento de insatisfacción y de desbordamiento por parte de las enseñantes francesas. Al final de nuestra entrevista, Diane, profesora agregada de inglés y madre de tres niños me explica a modo de confidencia :

"Pues te diré que hoy sueño con esto : pagar a alguien para que se ocupe de los niños el domingo mientras yo estoy allí mismo, en la habitación del al lado, disfrutando de un baño de espuma y leyendo un buen libro. Estoy considerándolo muy seriamente". Diane, 41 años, Francia

Las prácticas de ocio de sus colegas masculinos se distinguen en varios aspectos. En primer lugar, los enseñantes operan una "compartimentación" de los tiempos profesionales y personales. Además, dan fe de un acceso más amplio a tiempos de ocio individuales, que no están centrados sobre su familia. En repetidas ocasiones, tanto en Francia como en España, los hombres han subrayado el carácter incompresible y vital de su tiempo personal :

\footnotetext{
"Yo necesito mi tempo propio, no puedo renunciar a eso, siempre ha sido así, soy una persona bastante independiente, yo tengo mis amigos y... soy de un grupo ciclista, y muchas veces me voy fuera por la mañana; pero es, digamos, no puedo evitarlo, es mi salud mental, yo necesito salir con los amigos, reír, tomar unas cervezas, esto lo necesito". Pedro, 44 años, España
}

Varios hombres han confesado igualmente que rehúsan comprometerse más con sus actividades profesionales y adaptarse, por tanto, a las exigencias que supone la realización de "una buena carrera docente" (por ejemplo solicitando un puesto cuando se convocan oposiciones en Francia o formando parte del equipo de dirección en España - es el caso de Pedro). Nuestra población encuestada cuenta con varios casos de hombres, padres de varios hijos (uno de ellos tiene cinco y otros dos tienen tres) que practican muy intensamente actividades de ocio (ciclismo, música, judo...), mientras que mujeres asiduas a actividades de ocio similares explican cómo han tenido que abandonarlas con la llegada de su segundo hijo.

Si las prácticas de ocio de francesas y españolas se asemejan en algunos aspectos, la fuerte conciencia de género que demuestran las segundas constituye sin embargo un resorte para que las impulsa a desarrollar estrategias de negociación del "tiempo para sí mismas" con sus parejas, negociaciones que derivan a veces en fuertes tensiones. Hay que decir que estas españolas han tenido frecuentemente sus hijos más tarde que sus colegas francesas y poseen una experiencia más prolongada de vida en solitario (o en pareja) y de las prácticas de sociabilidad asociadas a ella. De hecho, los ocios individuales aparecen como elementos constitutivos de su identidad. En ese sentido, el discurso de Ana, profesora de música y madre de dos hijos de seis y ocho años respectivamente, es revelador :

"El hecho de ser los dos profesores tiene muchas ventajas, pero también tiene muchos inconvenientes... Es que te pasas muchas horas con los niños, y todo eso que las familias hoy en día buscan tanto y quieren, pero también 
hay un término medio, nosotros estamos mucho con ellos, entonces a nivel personal, te falta tu espacio y te falta hacer cosas para ti, y con eso nos hemos puesto muy en serio porque nos faltaba poder quedar con tus amigos, o poder hacer lo que quieras hacer durante un día, desde hacer deporte a irte al cine, lo que quieras". Ana, 39 años, España

La falta de tiempo para sí mismas es igualmente señalada por nuestras enseñantes españolas, y con mayor razón todavía cuando tienen hijos o asumen responsabilidades administrativas en su establecimiento escolar. Al hacerlo, la mayoría de ellas explica los esfuerzos realizados para preservarse algún tiempo de ocio. Siguiendo con las estrategias de compartimentación de los tiempos profesionales y familiares, reservarse un cierto margen para el ocio forma parte de la gestión temporal de las enseñantes españolas. El horario denso y sobrecargado de Cristina (41 años), que forma parte del equipo directivo de su establecimiento, tiene en cuenta su voluntad de estar, por un lado, disponible para su hija única en cuanto llega a casa y, por otro, de disponer de media jornada por semana para sus actividades personales : "Ya sea para pasear, para ir a ver una exposición o cualquier cosa...". Más emancipadas de las representaciones tradicionales del "bloque ideal madre/esposa" (Daune-Richard \& Haicault, 1985) y de las presiones que inducen a practicar actividades de ocio orientadas hacia la familia, manifiestan una mayor distancia frente a la "usurpación afectiva" de que son objeto las mujeres de las sociedades contemporáneas (Molinier, 2011) y se muestran relativamente ajenas a cualquier sentimiento de culpabilidad por el hecho de delegar y confiar sus hijos a otras personas, recurriendo a asistentes remuneradas. arrojar alguna luz sobre los fenómenos de deterioración o alteración de la salud ?

\section{La organización sexuada de los tiempos de actividad : ¿un haz de luz sobre las problemáticas de salud del personal docente?}

40 Los resultados presentados hasta aquí atestiguan la diferenciación sexuada de las experiencias temporales que existe en el seno de la enseñanza secundaria pública, una diferenciación que reviste formas más agudas en Francia que en España. Más aún, dan fe de la pertinencia heurística de tomar en cuenta la organización de las distintas actividades (profesionales y extra-profesionales) como clave de lectura de las condiciones subjetivas de la realización del trabajo de las enseñantes. La articulación compleja e invasiva entre el trabajo docente y el trabajo doméstico y los cuidados, así como la subsiguiente dificultad de encontrar "tiempo para sí mismo", pueden permitirnos captar la singularidad de las experiencias de salud y de desgaste en el trabajo de ambos sexos. Esa articulación podría explicar, por lo menos en parte, por qué varios estudios, llevados a cabo en diferentes países, constatan que las enseñantes se declaran víctimas de estrés en relativa mayor proporción que sus homólogos masculinos (Castro Posada, 2005 ; Houlfort \& Sauvé, 2010 ; Matud Aznar et al., 2002). 


\section{1 “Enseñantes al borde de un ataque de nervios" : ¿una impostura?}

41 Al término de este análisis se desprende una visión de la profesión docente mucho más matizada que la de una profesión armoniosa con la vida familiar y, más aún, con la vida de las mujeres. En efecto, la asignación de las enseñantes a las responsabilidades familiares no deja de tener consecuencias en cuanto a la sensación de desbordamiento, pero también de culpabilidad por lo que se refiere al rendimiento en cada una de las esferas, que gestionan como pueden, en la mayoría de los casos solas y de manera precipitada. Mantener el "equilibrio" entre los distintos tiempos vitales, supone a la vez gestionar y cautivar la atención de alumnos ruidosos y no siempre dóciles, estar presente cerca de sus hijos y disponible para su pareja y asegurar el orden doméstico del hogar. El "nivel" exigido resulta muy alto para estas mujeres que intentan ser eficaces en todos los ámbitos. Las características individuales, como el sexo, la edad o la disciplina que imparten, influyen sobre ese sentimiento. Nuestras encuestadas francesas y españolas se declaran, en efecto, más desbordadas que sus homólogos masculinos, tanto más cuanto tienen hijos e imparten disciplinas literarias o niveles elevados de enseñanza.

Además, la autonomía en la organización del tiempo de trabajo parece coincidir aquí con una mayor dificultad para gestionar los horarios sin sentirse desbordada. Ese es particularmente el caso en Francia, donde el talante profesional docente está impregnado de una visión que otorga una importancia notable a disponer de cierta "libertad" en la organización del tiempo de trabajo (Jarty, 2010). Se trata de mantener así el potencial de transmisión de los "saberes cultos" de expertos en diferentes disciplinas (Moreau, 2011): el trabajo realizado "fuera de las paredes" del establecimiento se valora de modo particular y representa una porción importante de las tareas docentes (Jarty, 2011).

La realidad cotidiana de ciertas enseñantes se aleja, por tanto, de las representaciones clásicas de la docencia como una profesión que permite, tanto en el plano organizativo como en el psicológico, una articulación perfecta de las distintas esferas de la vida social. De hecho, "La gestión ordinaria de la vida en pareja" (Haicault, 1984) puede revelarse difícil y agotadora, incluso para enseñantes supuestamente aventajadas en cuanto a la gestión de las esferas profesionales y familiares. Esa constatación abunda en los resultados obtenidos por Dominique Cau-Bareille $(2009,2011)$ en un análisis ergonómico sobre finales de carrera docentes. Poniendo el acento sobre los efectos negativos, principalmente en términos de salud, de la búsqueda permanente de un equilibrio entre trabajo asalariado, trabajo doméstico y cuidados, percibe ahí una de las causas que explican las interrupciones precoces de la carrera profesional entre las mujeres. Esta pista merece ser tomada tanto más en serio cuanto que, sobre un trasfondo de "malestar" en la profesión (Caroly \& Messing, 2011; Gonzales Faraco, 2002), la acumulación a lo largo de toda la vida de responsabilidades profesionales y extra-profesionales podría contribuir a reforzar la voluntad de retirarse prematuramente del mercado de trabajo o, cuando menos, de reducir la inversión subjetiva - es decir, el grado de compromiso personal - que los individuos aportan a su trabajo. Comprometerse y "encontrar un sentido" a su actividad profesional podría, en efecto, verse debilitado cuando las distintas esferas vitales se mezclan. 
como una "barrera" protectora (frágil, sin duda) frente al desbordamiento. Como diría Pascale Molinier, el trabajo profesional puede entonces "funcionar como otra escena" de la vida social (Molinier, 2009: 2). Por otra parte, estas prácticas de compartimentación se acompañan, tal como hemos visto, de una rentabilización casi sistemática de los tiempos intersticiales (durante los desplazamientos, sobre todo) para trabajar. Pero la intrusión del trabajo profesional en el seno de este espacio-tiempo podría suponer un problema, porque en él se mezclan, sin transición alguna, todos los tiempos de los distintos "roles" femeninos (trabajadora/madre/esposa, etc.). Esos tiempos intersticiales ya no desempeñan su papel de "preparación" para pasar de una actividad a otra, asumiendo un papel diferente (el de madre, el de enseñante, el de esposa, el de militante...). Amy Wharton (2004) daba cuenta de lo importante que es para las mujeres evacuar el trabajo y deshacerse de su influencia antes de volver a casa y hacerse cargo de otra actividad. El tiempo transicional contribuye a acrecentar la disponibilidad necesaria para cada actividad. Las españolas carecen de él con frecuencia.

Entretanto, el sentimiento de malestar y la dificultad para gestionar el trabajo remunerado y el no remunerado son raramente percibidos como el resultado de una relación social por parte de las mujeres entrevistadas. En particular, desde el punto de vista de las relaciones de género, el mantenimiento, o cuando menos los restos, de la dominación masculina son poco evocados como fenómeno explicativo de su desbordamiento. Si algunas tienen la sensación de que "se están aprovechando de ellas", lo perciben mucho menos como la consecuencia de la baja participación de sus parejas en las tareas domésticas y educativas que como una cierta fatalidad, cuando no como su propia responsabilidad. La relevancia de una lectura de tipo psicológico de los problemas se ve alimentada en el mundo docente, además, por la débil credibilidad social otorgada a la existencia de tensiones de articulación entre el trabajo remunerado y el no remunerado en el seno de la profesión. En este contexto, la prevalencia de un discurso que señala las debilidades individuales reduce las posibilidades de una toma en consideración de dichas tensiones por parte de la jerarquía profesional y, por lo tanto, al cabo, de transformación de las condiciones de trabajo (Loriol, 2005) y, de modo más general, de los principios organizadores del sistema "sexo/género".

\section{2 (Re)pensar las problemáticas de salud que plantean las experiencias de "múltiple actividad"}

Los análisis del fenómeno de saturación frente al desarrollo de la "múltiple actividad" en situación de trabajo (remunerado) proliferan (Bidet, 2011a). Un número creciente de profesionales se ve confrontado a la gestión simultánea de tareas heterogéneas y conflictivas, a ajustes de último minuto, a interrupciones repetidas en el trabajo (Datchary, 2011). Estos arbitrajes permanentes se constatan también en el grupo profesional docente, para el que la extensión de las responsabilidades comporta, en el quehacer cotidiano, una acumulación de diversas tareas que se entrecruzan (clases, correcciones, diseño de proyectos, seguimiento de alumnos con dificultades) (Caroly \& Messing, 2011 ; Jarty, 2011). La sobrecarga de solicitaciones que emana de las diferentes esferas (productivas y reproductivas), y que este artículo pone de relieve, conlleva igualmente un sobre-trabajo a través del cual podemos aprehender lo que realmente comportan las experiencias de "múltiple actividad" y su diferenciación sexuada. Los esfuerzos que suponen las transacciones, continuas y acumulativas, entre los entornos 
profesionales y personales sumergen a los individuos, y a las mujeres en particular, en un ritmo de actividades difícil de soportar.

Esta pista va en el mismo sentido que varios estudios. La retórica política creciente a favor de una mejor "conciliación" de los tiempos vitales insiste, en efecto, sobre su impacto notable por cuanto se refiere a la calidad de vida de los individuos en general y a la igualdad entre los sexos en particular (Silvera, 2002 ; Torns, 2005). Desde el punto de vista de las condiciones objetivas de trabajo y de los riesgos para la salud, una encuesta de 2007, realizada por la Fundación europea para la mejora de las condiciones de vida y de trabajo, establece una correlación entre una falta de equilibrio entre vida profesional y vida privada y la degradación de la salud física (afecciones musculares y óseas, dolores en la nuca) (Knecht \& Hämmig, 2008). La autonomía en el trabajo se presentaba entonces como un elemento que favorecía la "conciliación de los tiempos vitales". Pero, son más raras las investigaciones que muestran la otra cara de dicha autonomía desde el punto de vista de la salud psíquica y mental. En el caso de la enseñanza, la fuerte autonomía de que gozan los y las enseñantes francesas les permite un grado de presencia (objetiva) relativamente elevado junto a sus hijos. Eso permite sin duda que la mayoría de madres conserven un empleo de jornada completa (US, 2008). Pero, cuando, siempre y de manera casi exclusiva, recae sobre las mujeres el peso de las múltiples tareas domésticas y familiares, así como la fatiga mental que ello supone, y cuando eso es así porque se supone que gracias a su profesión disponen del tiempo necesario para hacer frente a sus responsabilidades en todos los frentes, ¿no tendrá acaso ese compromiso múltiple una incidencia concreta en términos de cansancio ? Los resultados presentados en el marco de este artículo sugieren el carácter nefasto y poco (re)conocido de la interpenetración de los distintos "espacios-tiempos" que singulariza el ritmo del trabajo de las enseñantes francesas - más aún que el de sus homólogas españolas $\mathrm{y}$, sobretodo, más que el de sus colegas masculinos de ambos países. El peligro de instilación de una fatiga crónica, de una "pérdida de sentido del trabajo", podría aparecer con tanta mayor fuerza cuanto que el tiempo para reencontrase a sí misma (el llamado "tiempo para sí misma") se encuentra comprimido, reducido a su mínima expresión, o incluso se volatiliza.

Los contrastes observados entre Francia y España confirman la pertinencia heurística del método comparativo en el análisis del trabajo y de sus condiciones de realización. La adaptabilidad más concreta de los entornos profesionales españoles (como el hecho de disponer de un espacio tranquilo para adelantar el trabajo en el mismo seno del establecimiento), pero también la propia concepción de la profesión, que empuja menos que en Francia a los alardes académicos y, por lo tanto, no supone una presión incesante para estar siempre "al más alto nivel", parecen contribuir a una indiferenciación progresiva de las prácticas de ambos sexos. A partir de ahí, no se trata de afirmar sin embargo la desaparición de las relaciones de género y de poder en el marco de la docencia española (ni tampoco - o quizás menos todavía - en el contexto nacional español). Varios indicadores dan fe de su persistencia: la sobreexplotación, por parte de las enseñantes, de una mano de obra femenina poco cualificada, mal remunerada, con frecuencia inmigrada (Peterson, 2007 ; Torns, 2008) para descargarse lo más posible del tiempo de trabajo doméstico, constituye un ejemplo de ello; la desigual distribución de hombres y mujeres en la jerarquía docente, otro (Instituto de la Mujer, 2001 ; Grañeras Pastrana \& Saval Ceres, 2004) ; como también lo sería el menor acceso de las mujeres al "tiempo para sí" en comparación con sus homólogos 
masculinos. Pero la compartimentación de las diversas esferas sociales establecida en España parece más propicia a una cierta equiparación de las condiciones objetivas y subjetivas de realización del trabajo.

\section{Conclusión}

En la enseñanza secundaria francesa y española, la obligación de articular la vida profesional y la vida familiar que aún pesa mayoritariamente sobre las mujeres aparece como una explicación mayor de la diferenciación sexuada en la organización del tiempo de trabajo. Sin duda, el ritmo del trabajo docente está íntimamente ligado al talante profesional dominante en cada contexto social. No obstante, ese ritmo sigue marcado por el género y, aunque esta diferenciación sexuada se expresa de modo distinto en el caso de las francesas y en el de las españolas, sus experiencias tienden a converger en una acumulación de tareas, la múltiple actividad.

Así pues, más allá de las presiones ligadas a un trabajo exigente a las que se ve confrontado el personal de ambos sexos en términos de gestión del ruido y de "alarde profesional" en clase, de incremento de las tareas y de compromiso emocional con los alumnos, o incluso de implicación en universos profesionales no siempre adaptados (Caroly \& Messing, 2011; Riel \& Messing, 2011), el peso de las condiciones extraprofesionales de realización del trabajo parece engendrar un estrés suplementario que se inscribe de manera específica en las experiencias de las mujeres.

51 Para ellas, la dificultad de construir una separación impermeable entre vida profesional y familiar en el seno de una profesión del conocimiento impregnada de relaciones emocionales a veces intensas (Lantheaume \& Helou, 2008) lleva a pensar que las relaciones con el trabajo no son idénticas en función de la pertenencia sexuada. Mostrar la fragmentación y el solapamiento de actividades que emanan de las esferas públicas y privadas significa también brindar unas claves de lectura indispensables para comprender las experiencias profesionales de las mujeres y sus repercusiones por cuanto a la salud se refiere. Tal como nos enseña la situación en España, la instauración de una criba de las diversas temporalidades sociales aparece como el medio de evitar, por lo menos en parte, el desgaste y el sufrimiento asociados a la superposición y a la dispersión de los "espacios-tiempos". Semejante fenómeno resulta tanto más problemático cuanto que es mal (re)conocido e inconfesable en una profesión en que esos rasgos penosos se suponen poco importantes y apenas son tomados en cuenta por las organizaciones. En ese sentido, existe la necesidad imperiosa de nombrar y reconocer la múltiple actividad de las enseñantes, sin perder de vista la dimensión macro-social de un cuestionamiento de la división sexual del trabajo. Pues, la igualdad de los sexos en el trabajo se dirime a varios niveles. 


\section{BIBLIOGRAFÍA}

Almudever, B., Croity-Belz, B., Hajjar, V., \& Fraccaroli, F. (2006). Conditions for efficiency of the self-efficacy beliefs in regulating professional disruption : dynamics of the system of Activities. Psychologie du travail et des organisations,12, 151-166.

Astelarra, J. (2005). Veinte años de políticas de igualdad, Madrid : Ediciones Cátedra.

Barrere-Maurisson, M.-A. (2004). Egalité hommes-femmes et parentalité. Où va-t-on ? - avec la participation de Sabine RIVIER, La lettre du MATISSE. Retiré de http://matisse.univ-paris1.fr/ barrere, 8 mars 2004, 4 pages.

Bidet, A. (2011a). La multiactivité, ou le travail est-il encore une expérience ? Communications, 89 Travailler, 9-26.

Bidet, A. (2011b). L'engagement dans le travail. Qu'est-ce que le vrai boulot ? Paris : PUF.

Burton-Jeangros, C., \& Camenisch, M. (2006). Etat de santé, situation familiale et activité professionnelle. La double charge est-elle mauvaise pour la santé des femmes ? Newsletter du Panel suisse de ménages, Printemps, 1-4.

Butt, G., \& Lance, A. (2005). A Secondary school teacher workload and job satisfaction, do successful strategies for change exist ? Educational Management Administration and Leadership, 33, 401-422.

Cacouault-Bitaud, M. (2003). La sociologie de l'éducation et les enseignants : cherchez la femme... In Jacqueline Laufer, Catherine Marry e Margaret Maruani (Eds.), Le travail du genre (pp. 163-180). Paris : La Découverte.

Cacouault-Bitaud, M. (2007). Professeurs... mais femmes. Carrières et vies privées des enseignantes du secondaire au XXème siècle. Paris : La Découverte.

Caroly, S., \& Messing, K. (2011). Introduction : Gender, work schedules and work/family regulation. Work, 40, (5), 1-3. Disponible en ligne, consulté le 15 janvier 2012 : http:// www.metapress.com/content/7173821k307110r2/fulltext.pdf

Carrasquer Oto, P., Torns, T., Tejero, E., \& Romero, A. (1998). El trabajo reproductivo. Papers : Revista de sociología, 55, 95-114.

Castro Posada, J.-A. (2005). El estrés laboral en el profesorado femenino y masculino de E.S.O. y Bachillerato : sus dimensiones, su relación con otros factores psicológicos, y valoración de la eficacia de un procedimiento de intervención con seguimiento. Madrid : Instituto de la mujer.

Cau-Bareille, D. (2009). Les fins de carrières en maternelle. Rapport interne du CREAT : Centre d'Etudes et de l'emploi.

Cau-Bareille, D. (2011). Factors influencing early retirement in a female-dominated profession : Kindergarten teacher in France, Work, 40, (5), 15-30.

Connell, R.W. (1987). Gender and power. Cambridge : Polity Press.

Curie, J. (2002). Parcours professionnels et interdépendances des domaines de vie. Revue Éducation Permanente, 150, 23-32.

Datchary, C. (2011). La dispersion au travail. Toulouse : Octarès. 
Daune-Richard, A.-M., \& Haicault, M. (1985). Le poids de l'idéel dans les rapports sociaux de sexe, Cahiers de l'APRE, $n^{\circ} 3,49-91$.

Deem, R. (1986). All Work and No Play? The Sociology of Women and Leisure. Open University Press : Milton Keynes.

Duran, M.-A. (2006). El valor del tiempo. ¿Cuántas horas te faltan al día ? Madrid : España.

ETUCE. (2011). Teacher's work related stress European-wide Survey - Assessment, Comparison and Evaluation of the Impact of Psychosocial Hazards on Teachers at their Workplace in the EU. Disponible en ligne, http://etuce.homestead.com/Publications2011/WRS_Brochure_Final_ENG_doc.pdf

Fernandez Cordon, J.-A., \& Tobio Soler, C. (2005). Conciliar las responsabilidades familiares y laborales : Políticas y prácticas sociales. Documento de Trabajo, 79. Fundación Alternativas. Disponible en ligne, http://www.pid.evimo.es/ConciliacionFamiliarMadres.pdf

Fusulier, B. (2011). Articuler vie professionnelle \& familiale. Etude de trois groupes professionnels : les infirmières, les policiers et les assistants sociaux. Louvain-la-Neuve : Presse Universitaire de Louvain.

Gadbois, C. (1975). L'analyse des emprises réciproques de la vie de travail et de la vie hors travail. Bulletin du CERP, 23, (2), 117-151.

Gonzalez Faraco, J.-C. (2002). Entre modernisation et nostalgie. La fonction enseignante et les réformes scolaires en Espagne. Revue Internationale d'Education de Sèvres, 30, 69-83.

Grañeras Pastrana, M., \& Saval Ceres, J. (2004). Mujeres en cargo de representación en el sistema educativo. Madrid : Instituto de la Mujer.

Haicault, M. (1984). La gestion ordinaire de la vie à deux. Sociologie du Travail, 3, 268-277.

Heinen, J., Hirata, H., \& Pfefferkorn, R. (2009). Politiques publiques et articulation vie professionnelle / vie familiale. Cahiers du Genre, 46, 5-16.

Houlfort, N. \& Sauvé, F. (2010). Santé psychologique des enseignants de la Fédération autonome de l'enseignement. Montréal, Québec : École nationale d'administration publique.

Instituto de la Mujer - CIDE (2001). Las mujeres en el sistema educativo. Madrid : Instituto de la Mujer.

Jarty, J. (2009). Les usages de la flexibilité temporelle chez les enseignantes du secondaire. Temporalités, 9 [En ligne] : http://temporalites.revues.org/1057

Jarty, J. (2010). L'emploi, la famille et l'articulation des temps de vie chez les enseignant.e.s du secondaire. Une comparaison France-Espagne. Thèse de sociologie, Université de Toulouse Le Mirail, Université Autonome de Barcelone.

Jarty, J. (2011). Le travail invisible des enseignants “ hors les murs ». Ethnographiques.org, 23, Analyser les présences au travail : visibilités et invisibilités. Disponible en ligne, http:// www.ethnographiques.org/2011/Jarty

Junter-Loiseau, A. (1999). La notion de conciliation de la vie professionnelle et de la vie familiale. Révolution temporelle ou métaphore des discriminations ? Cahiers du Genre, 24, 73-98.

Kergoat, D. (2000). Division sexuelle du travail et rapports sociaux de sexe. In Héléna Hirata, Françoise Laborie, Hélène Le Doaré et Danièle Sénotier (Eds.), Dictionnaire critique du féminisme (pp 35-44). Paris, Presse Universitaire de France, 35-44.

Knecht, M., \& Hämmig, O. (2008). Manque d'équilibre entre vie professionnelle et vie privée et troubles affectant le dos. Résultats choisis d'une enquête en entreprise de grande ampleur. Zurich : EPF Zurich, Rapport au Département fédéral de l'économie (DFE), Secrétariat d'Etat à l'économie (SECO). 
Lantheaume, F., \& Helou, C. (2008). La souffrance des enseignants. Une sociologie pragmatique du travail enseignant. Paris : PUF.

Le Feuvre, N. (2007). Les processus de féminisation au travail : entre différenciation, assimilation et "dépassement du genre". Sociologies pratiques, $n^{\circ} 14,11-15$.

Le Feuvre, N. (2010). Feminising professions in Britain and France : How countries differ. In Jacqueline Scott, Rosemary Crompton et Clare Lyonette (Eds.), Gender inequalities in the 21st century: New barriers and continuing constraints (pp. 126-149). London : Edward Elgar.

Le Feuvre, N. (2011). Les enjeux de santé au travail chez les seniors des deux sexes : Du pareil au même ? In Sophie Le Garrec (Dir.), Le travail contre la santé ? Les enjeux de santé au travail des seniors (pp. 119-146). Paris : l'Harmattan.

Letablier, M.-T. (2009). Travail et parentalité : des régimes de conciliation variables en Europe. In Chantal Nicole-Drancourt (Dir.). Conciliation travail-famille. Attention travaux (pp. 77-87). Paris : L'Harmatthan.

Loriol, M. (2005). Les ressorts de la psychologisation des difficultés au travail. Une comparaison entre infirmières, policiers et conducteurs de bus. Cahiers de recherches sociologiques, $n^{\circ} 41-42$, 191-208.

Marry, C. (2004). Femmes ingénieures, Une révolution respectueuse. Paris : Belin.

Matud Aznar, M.-P., Garcia Rodriguez, \& Matud Aznar, M.-J. (2002). Estrés laboral y salud en el profesorado : un análisis diferencial en función del género y del tipo de enseñanza. Revista Internacional de Psicología Clínica y de la Salud, 2, 3, 451-465.

Meda, D., \& Perivier, H. (2007). Le deuxième âge de l'émancipation. La société, les femmes et l'emploi. Paris : Le Seuil.

Messing, K., \& Östling, P. (2006). Gender Equality, work and health - A Review of the Evidence. Geneva : World Health Organisation.

Molinier, P. (2009). Temps professionnel et temps personnel des travailleuses du care: perméabilité ou clivage? Temporalités 9. Disponible en ligne, http://temporalites.revues.org/ index988.html

Molinier, P. (2011). Interview : Les femmes doivent apprendre l'égoïsme, Journal des femmes. Disponible en ligne, http://www.journaldesfemmes.com/maman/0409bureau_maison/ 2interview.shtml

Moreau, M.-P. (2011). Les enseignants et le genre. La construction des inégalités sexuées dans l'enseignement du second degré en France et en Angleterre. Paris : PUF.

Perrot, M. (1987). Métiers de femmes. Mouvement social, 140, 3-8.

Peterson, E. (2007). The invisible carers : Framing domestic work(ers) in gender equality. European Journal of Women's Studies, 14, (3), 265-280.

Riel, J., \& Messing, K. (2011). Counting the minutes : Administrative control of work schedules and time management of secondary school teachers in Québec. Work, 40, (5), 59-70.

Rovira, M. (1993). Les dones ensenyants. Una situacio de desigualtat. In Montserrat Rovira \& Amparo Tomé (Dirs.), L'ensenyament, una professio femenina? (pp. 13-91). Barcelona : Institut de Ciències de l'Educacio de la UAB.

Silvera, R. (2002). Articuler vie familiale et vie professionnelle en Europe. Un enjeu pour l'égalité. Paris : La Documentation Française. 
Teiger, C. (1980). Les empreintes du travail. In Société française de psychologie du travail (Dir.), Équilibre ou fatigue par le travail ? (pp. 25-44). Paris : Entreprise Moderne d'édition.

Tobio Soler, C. (2005). Madres que trabajan: Dilemas y estrategias. Madrid : Cátedra.

Torns, T. (2005). De la imposible conciliación a los permanentes malos arreglos. Cuadernos de Relaciones Laborales, 23, (1), 15-33.

Torns, T. (2008). El trabajo y el cuidado : Cuestiones teórico-metodológicas desde la perspectiva de género. Empiria : Revista de Metodología de Ciencias Sociales, 15, 53-73.

Torns, T., \& Moreno, S. (2008). La conciliación de las jóvenes trabajadoras : Nuevos discursos, viejos problemas. Revista de Estudios de Juventud, 83, 101-117.

US. (2008). Fichier de paye des Trésoreries générales au 31 janvier 2005. L'US supplément, $\mathrm{n}^{\circ}$ 663, 23.

Wharton, A.S. (2004). Femmes, travail et émotions : concilier emploi et vie de famille. Travailler, 12, 135-160.

\section{NOTAS}

1. En 2008 , había respectivamente $57^{\prime} 8 \%$ de enseñantes de secundaria en Francia y $57 \%$ en España (Fuente : Ministère de l'Education Nationale y Ministerio de Educación).

2. La utilización del término "crisis" de la profesión se refiere menos, como lo subrayaron Lantheaume y Hélou (2008), a su definición corriente, tendente a sugerir un malestar de tipo regresivo, que a un contexto inestable de redefinición de los referentes profesionales y las finalidades del trabajo docente.

3. La población encuestada en Francia se compone de 25 mujeres, tres de las cuales ocupan actualmente puestos de dirección en sus establecimientos escolares, y 15 hombres - dos de ellos en puestos de dirección. La población española encuestada cuenta, por su parte, con 20 mujeres y 15 hombres, de los cuales la mitad formó parte o sigue integrada en un equipo de dirección. Las entrevistas se desarrollaron en la lengua del país de origen de las personas entrevistadas, es decir en francés y español, excepto en el caso de algunos españoles que enseñan francés. En esos casos particulares, las entrevistas se realizaron en francés o alternando las dos lenguas.

4. Esas actividades son ampliamente delegadas por los y las enseñantes francesas a los personales de dirección o de educación, dos cuerpos profesionales que, contrariamente a lo que ocurre en España, desempeñan misiones netamente distintas de las del cuerpo docente.

5. Hemos podido constatar, sin embargo, diferencias nada despreciables según los establecimientos y la antigüedad de los enseñantes en cada centro, de tal modo que la negociación de los horarios podía en algunas ocasiones revelar lógicas de poder entre los y las enseñantes o bien entre enseñantes y personal de dirección.

\section{RESÚMENES}

Este artículo apunta a esclarecer los mecanismos de diferenciación sexuada referidos a las prácticas de organización del trabajo en el seno de una profesión cuyo nivel de estrés se revela 
particularmente elevado: la enseñanza de segundo grado. Este estudio establece una comparación entre Francia y España, dos países cuyas normas profesionales en materia docente difieren y conllevan compromisos singulares al trabajo.

Apoyándose sobre los resultados de una encuesta cualitativa, la autora concede una importancia central a los discursos y representaciones que los individuos desarrollan acerca de sus experiencias profesionales.

La consideración de los distintos marcos de trabajo (profesionales y extra-profesionales) permite formular una hipótesis : a pesar de no traducirse del mismo modo en los dos países estudiados, los acomodamientos espacio-temporales sexo-diferenciados tienen repercusiones en lo que a la salud se refiere, especialmente en términos de estrés. Sin embargo, la sensación de "desbordamiento" frecuentemente detectada constituye una percepción mal (re)conocida, e incluso públicamente inconfesable en una profesión recubierta de un aura family friendly.

Este artigo visa dar visibilidade aos mecanismos de diferenciação sexuada no que diz respeito às práticas de organização do trabalho no seio de uma profissão em que o nível de stresse se revela particularmente elevado : o trabalho docente no(s) ciclo(s) de ensino destinado(s) a crianças com uma idade superior aos 11 anos. Neste âmbito, é estabelecida uma comparação entre França e Espanha, dois países cujas normas profissionais de ensino diferem entre si e convocam um compromisso singular no trabalho.

Apoiando-se nos resultados de uma pesquisa qualitativa, a autora atribui uma importância central aos discursos e representações que os indivíduos sustentam sobre as suas experiências profissionais. A consideração dos diferentes contextos de trabalho (profissionais e extraprofissionais) permite avançar uma hipótese : embora não se traduza da mesma forma nos dois países, as estratégias espacio-temporais sexo-diferenciadas têm consequências do ponto de vista das experiências no plano da saúde, nomeadamente em termos de stresse. Todavia, a sensação de "sobrecarga", frequentemente identificada, permanece mal (re)conhecida, e até mesmo publicamente inconfessável, numa profissão considerada family friendly.

Cet article vise à éclairer les mécanismes de différenciation sexuée des pratiques d'organisation du travail au sein d'une profession où le niveau de stress est particulièrement élevé : l'enseignement du second degré. Il prend pour point de comparaison la France et l'Espagne, deux pays où les normes professionnelles enseignantes différent et engendrent un engagement singulier dans le travail.

S'appuyant sur les résultats d'une enquête qualitative, l'auteure accorde une importance centrale aux discours et représentations que les individus posent sur leurs expériences au travail.

La prise en compte des différents cadres de travail (professionnels et extra-professionnels) permet d'étayer une hypothèse : sans se traduire de la même manière dans les deux pays, les arrangements spatio-temporels sexo-différenciées ont des conséquences du point de vue des expériences de santé, notamment en termes de stress. Toutefois, le sentiment de "débordement", fréquemment identifié, demeure mal (re)connu, voire publiquement inavouable dans une profession réputée family friendly.

This paper seeks to clarify the mechanisms of gender-based differentiation in everyday practices of work organization within a profession where employees face a particularly high level of stress : secondary school teaching. It compares France and Spain, two countries where the professional teaching standards differ, giving rise to a particular spatio-temporal involvement at work.

Focusing on the results of a cross-national qualitative survey, the author gives prime importance to the speeches and representations that individuals use to describe their experiences at work. Taking into account the various working environments (career-related and non career-related), it leads us to observe that, without translating in the same way within the two countries studied, 
gender-differentiated spatial and temporal arrangements have ramifications from a health point of view. However, the identified feeling of "overload" is often going unrecognised, or even being officially disavowed, within this profession with a family-friendly reputation.

ÍNDICE

Keywords: teaching work, gender, work/life balance, health at work, cross-national comparison Mots-clés: travail enseignant, genre, articulation travail/famille, santé, comparaison FranceEspagne

Palavras-chave: trabalho docente, género ; articulação trabalho/família, saúde, comparação França/Espanha

Palabras claves: trabajo docente, género, articulación trabajo/familia, salud, comparación Francia-España

\section{AUTOR}

\section{JULIE JARTY}

Université de Lausanne, Centre en Études Genre, Bâtiment Anthropôle, Quartier UNIL Dorigny, 1015 Lausanne, Suisse

Julie.Jarty@unil.ch 